\title{
Article \\ Effect of Soccer Ball Panels on Aerodynamic Characteristics and Flow in Drag Crisis
}

\author{
Yuki Sakamoto ${ }^{1}$, Masaki Hiratsuka ${ }^{2}$ (D) and Shinichiro Ito ${ }^{2, *(\mathbb{D}}$ \\ 1 Department of Mechanical Engineering, Graduate School of Kogakuin University, 1-24-2 Nishi-Shinjuku, \\ Shinjuku-ku, Tokyo 163-8677, Japan; am19022@ns.kogakuin.ac.jp \\ 2 Department of Mechanical Engineering, Faculty of Engineering, Kogakuin University, 1-24-2 Nishi- Shinjuku, \\ Shinjuku-ku, Tokyo 163-8677, Japan; hiratsuka@cc.kogakuin.ac.jp \\ * Correspondence: ito@cc.kogakuin.ac.jp; Tel.: +81-90-8461-0487
}

check for updates

Citation: Sakamoto, Y.; Hiratsuka, M. Ito, S. Effect of Soccer Ball Panels on Aerodynamic Characteristics and Flow in Drag Crisis. Appl. Sci. 2021, 11, 296. https://doi.org/10.3390/ app11010296

Received: 12 November 2020 Accepted: 16 December 2020 Published: 30 December 2020

Publisher's Note: MDPI stays neutral with regard to jurisdictional clai$\mathrm{ms}$ in published maps and institutional affiliations.

Copyright: (C) 2020 by the authors. Licensee MDPI, Basel, Switzerland. This article is an open access article distributed under the terms and conditions of the Creative Commons Attribution (CC BY) license (https:// creativecommons.org/licenses/by/ $4.0 /)$.

\section{Featured Application: Soccer balls.}

\begin{abstract}
The panel patterns of soccer balls that change with each World Cup have a significant impact on the balls' aerodynamic and flight characteristics. In this study, the aerodynamic forces of eleven types of soccer ball with different panel patterns were measured in a wind tunnel experiment. We characterized the panel shapes of soccer balls by the length, cross-sectional area, and the panel grooves' volume. The results confirmed that the drag and drag crisis characteristics are dependent on the groove length and volumes. Flow separation points were visualized by an oil film experiment and particle image velocimetry (PIV) measurement to understand the drag crisis of the soccer balls. The results showed that the panel shape of the ball significantly changes the position of the separation point near the critical region, where the drags crisis occurs. In the critical region, laminar and turbulent flows coexist on the ball. On the other hand, the effect of panel shape on the separation point position is small in subcritical and supercritical states.
\end{abstract}

Keywords: drag crisis; soccer ball; flow separation; oil film; particle image velocimetry (PIV)

\section{Introduction}

In soccer, the aerodynamic characteristics of the ball have a significant effect on the development of the game. A new type of soccer ball is introduced at each World Cup and other major international tournaments, and the shape of the panels that make up the ball has a significant influence on its behavior. The Teamgeist, the ball used in the 2006 World Cup, was made up of sandpaper-shaped panels instead of the traditional pentagonal and hexagonal ones. Teamgeist's flight path changes irregularly when it is kicked in a way that does not allow the ball to rotate, and many impressive goal scenes were created by using this blur ball. This technique attracted people all over the world and became a new style of soccer. After this period, the ball's surface panels became diversified, and balls with various types of panels, such as star-shaped and vortex-shaped balls, appeared. The influence of the panel shape on the ball's flight path has become an essential aspect of ball design. Various aerodynamic studies have been conducted to elucidate the mechanism of this effect.

When the ball is in drive spinning, the drag and lift due to the Magnus force are on the ball $[1,2]$. The magnitude of the lift force is greatly affected by the speed and rotation rate of the ball. The negative Magnus force is also reported under limited conditions [3]. On the other hand, the irregular lateral forces that cause blurring balls were not measured in the drive spin ball, so irregular flights were not observed.

In contrast, when the ball does not spin, a lateral force is applied to the ball that causes irregular flight paths. In a non-spinning ball, twin vortices are formed in the wake of the ball, and jets between the vortices cause the lateral force. The presence of twin vortices 
and jets in the wake of the ball has been confirmed by visualization measurements using smoke [4] and tuft grids [5]. Synchronized measurements of the lateral force and the wake vortex using 3D-PIV (three-dimensional Particle Image Velocimetry) showed that, the jet was the cause of the lateral force. The direction and flow rate of the formed jet coincided with the lateral force applied to the ball [6].

It was also reported that for the balls composed of asymmetric panels with low gyroscopic rotation, the jet's direction formed in the wake changes with the ball's rotation [7]. On the other hand, no significant change in jet direction was observed when the panel shape was symmetrical. These results suggest that the panel shape is related to the degree of change in the jet flow direction and the resulting blur in the flight path.

In addition to the lateral force, the magnitude of the drag force on non-spinning balls varies greatly between different ball panels that has a significant effect on the flight trajectory [8,9]. In particular, it has been reported that the length of the ball's seam and the cross-sectional area and depth of the ball affect lift and drag $[9,10]$, and there is a relationship between the smaller lateral forces in balls with shorter groove lengths [11,12].

The Reynolds number in which the drag crisis occurs is also affected by the number of grooves in the ball $[13,14]$. The drag crisis is a phenomenon in which the drag coefficient decreases significantly as the flow changes from laminar to turbulent, increasing the Reynolds number. Dimpled balls also induce drag crisis at a smaller Reynolds number than those without [15]. The region of the Reynolds number where the flow transitions from laminar to turbulent is called the critical region, which is a complex flow between laminar and turbulent flows. Immediately after the kick, the velocity of the soccer ball is so high that the surrounding flow becomes turbulent. However, when the velocity decreases due to drag, the ball passes through this critical region and reaches the laminar flow region. Therefore, it is important to understand the flow around the ball in the critical region to consider the ball's trajectory [16-18].

Visualization of the flow is one of the most effective methods to elucidate the mechanism of the drag and lateral forces applied to the ball. Besides the previous visualization measurements on the jet flow [4-6], Hong et al. $[19,20]$ reported that the position of flow detachment varied with the location of the ball panel grooves, the number of seams, and the spacing of the grooves, using the 2D particle image velocimetry (PIV) measurement. Visualization measurements of flow using smoke wire reported that the flow separation position moved back further for balls with larger surface roughness than balls with smaller surface roughness [3].

However, previous visualization measurements have mainly focused on the laminar and turbulent regions. The critical region, which is considered important in twin vortices and irregular orbital changes, is still unexplored. In this study, we visualized and measured the effects of panel type and ball orientation on the flow and the ball's flight path in the critical region. First, drag coefficients were measured for 11 different types of soccer balls. Next, we measured the separation points of the flow in the critical region by oil film experiments and 2D-PIV measurements for selected balls.

\section{Materials and Methods}

The following four types of experiment were conducted in this study: measurement of the groove shape to characterize the surface properties of the balls, fluid force measurements, oil film experiments to identify the location of flow separation, and 2D-PIV measurements to measure the flow in the vicinity of the separation location. The eleven balls used in the experiments are summarized in Table 1. All of the balls were employed in past international competitions. It can be observed that the number and shape of the panels that make up the ball vary greatly. All of the balls have a circumference of 0.068 $0.070 \mathrm{~m}$, a diameter of $0.22 \mathrm{~m}$, a weight of $410-450 \mathrm{~g}$, and a gauge pressure of $0.06-0.11 \mathrm{MPa}$, which meets the standards of the FIFA (International Federation of Association Football). Groove shape and fluid force measurement were performed on all balls, but the oil film 
experiments and 2D-PIV measurements were applied on only a few of the balls listed in Table 1.

Table 1. A list of the balls measured in this study. Groove and aerodynamic force measurements were performed on all the balls. Oil film and 2D-PIV measurements were performed on the balls marked with a circle in the table.

\begin{tabular}{|c|c|c|c|c|c|c|}
\hline Name & Merlin & Telstar18 & Krasava & Ordem & Finale & Vantaggio \\
\hline Design & & & & & & vantraggiv \\
\hline Oil film & & 0 & $\bigcirc$ & & $\bigcirc$ & 0 \\
\hline 2D-PIV & & & & & & 0 \\
\hline Name & Evopower & Delta & Tango12 & Jabulani & Teamgeist & \\
\hline Design & & & & & & \\
\hline \multicolumn{7}{|l|}{ Oil film } \\
\hline 2D-PIV & 0 & & & 0 & 0 & \\
\hline
\end{tabular}

\subsection{Groove Shape Measurement}

To characterize the balls by the shape of the groove, the length, cross-sectional area, and total volume of the grooves at the panel joint of each ball were measured. The groove length was measured by placing a string over all the grooves. Some soccer balls have more than one type of panel binding groove. Each type of groove was measured three times, and its average value was determined. $3.6 \%$ of the maximum variation for each ball in the three measurements. The cross-sectional area and dimensions of the grooves were measured using a KEYENCE VR-3000 one-shot 3D-shape instrument, as shown in Figure 1. The cross-sectional area and depth of the grooves were calculated using the application, as shown in Figure 2. The depth and cross-sectional area were determined with an accuracy of $1.00 \times 10^{-3} \mathrm{~m}$ and $1.00 \times 10^{-6} \mathrm{~m}^{2}$, respectively. The total groove volume was calculated by multiplying the groove length by the cross-sectional area for each groove type. The total groove volume was defined by the following equation:

$$
V=\sum_{i=1}^{n} L_{i} \cdot S_{i}
$$

wheres $V$ shows the total groove volume of a ball, $L_{i}$ shows the length of $i$ th type of groove, $S_{i}$ shows the cross-sectional area of $i$ th type of groove, and $n$ shows the number of groove types in a ball. 


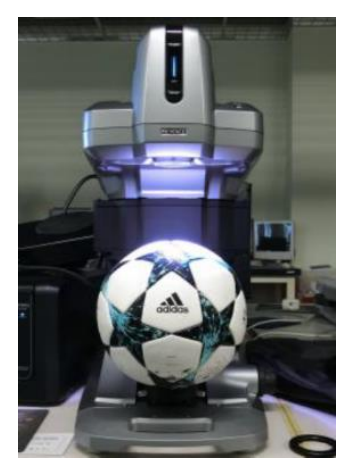

Figure 1. Groove shape measurement.

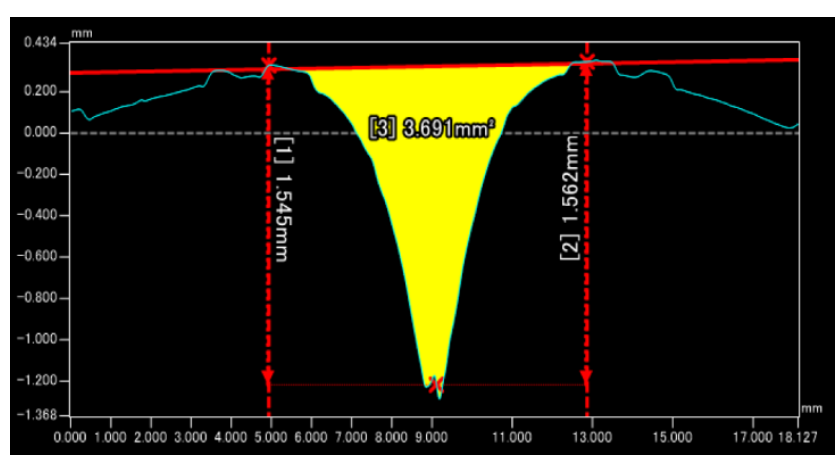

Figure 2. An example of groove shape measurement. The green line shows the shape of the ball surface, the red line indicates the depth of the groove, and the yellow area denotes the cross section of the groove.

\subsection{Fluid Force Measurement}

Fluid force measurements were performed on eleven types of different soccer balls with a wind tunnel. The wind tunnel we used is an open type with a sirocco fan. The outlet's size is $0.38 \mathrm{~m} \times 0.38 \mathrm{~m}$, and the length of the measurement section is $0.78 \mathrm{~m}$. The velocity range was $3 \sim 29 \mathrm{~m} / \mathrm{s}$ with $\pm 1.5 \%$ fluctuation, and the turbulence intensity was $\pm 1.0 \%$. The fluid force was measured in the range of wind speed from 3 to $29 \mathrm{~m} / \mathrm{s}$ every $1 \mathrm{~m} / \mathrm{s}$. This speed range covers most of the speed of a soccer ball that is kicked in a real game [16]. For shooting or a free kick, the ball is kicked out at about $30 \mathrm{~m} / \mathrm{s}$, and aerodynamic drag decelerates it to about $15 \mathrm{~m} / \mathrm{s}$ [9]. The ball may also be kicked out at a slower speed when passing. The drag coefficients were measured by placing the ball so that the wind hit the upper panel face of the pictures shown in Table 1. The force acting on the soccer balls was measured by a three component load cell $(F x, F y, F z)$, LMC-3520A by Nissho Electric Works. The measured values were sampled at $1000 \mathrm{~Hz}$ for $9.0 \mathrm{~s}$ and averaged. The experimental conditions are shown in Figures 3 and 4 . The ball was mounted with cobra-type rear support. The drag coefficient, $C_{D}$, was defined by the following equation:

$$
C_{D}=\frac{D}{\frac{1}{2} \rho U^{2} \cdot A}
$$

where $D, r, U$, and $A$ denote the drag force of the ball, the density of the air, the uniform wind speed, and the projected cross-sectional area of the ball, respectively. Reynolds number $(R e)$ is a dimensionless number defined as the ratio of inertial force to viscous force. Reynolds number, $R e$, is defined as the following equation:

$$
R e=\frac{U \cdot D}{v}
$$

where $v$ denotes the kinematic viscosity. 


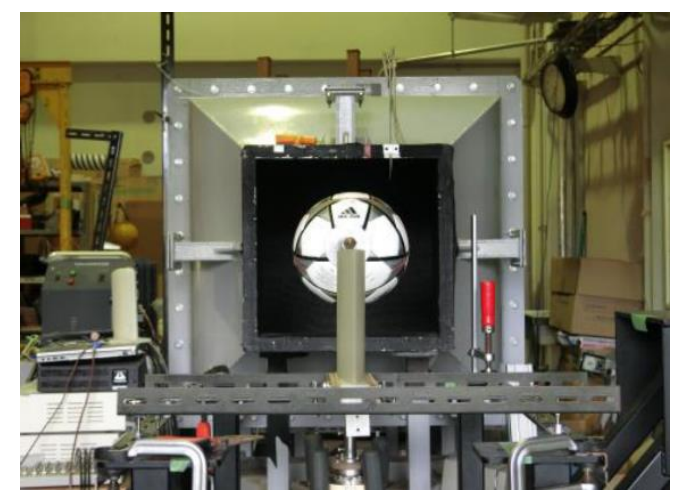

Figure 3. Fluid force measurement (back).

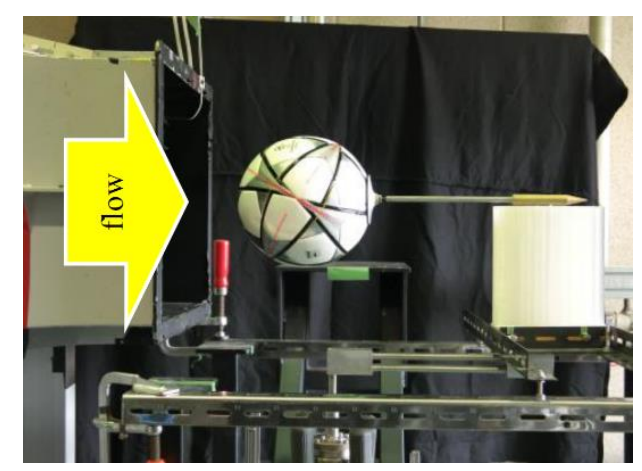

Figure 4. Fluid force measurement (side).

\subsection{Measurement of Separation Point by Oil Film Experiments}

In this method, oil film is applied to the ball surface under the wind flow. Since the oil film flows to the point where the flow separates from the ball surface, the separation point can be obtained by measuring oil distribution after measurement. As shown in Figure 5, the oil-film-coated ball was placed in the wind tunnel and measured by flowing the wind under a turbulent wind speed of $29.6 \mathrm{~m} / \mathrm{s}$. A mixture of oleic acid, liquid paraffin, and tetra-tri-iron oxide powder was employed for the oil.

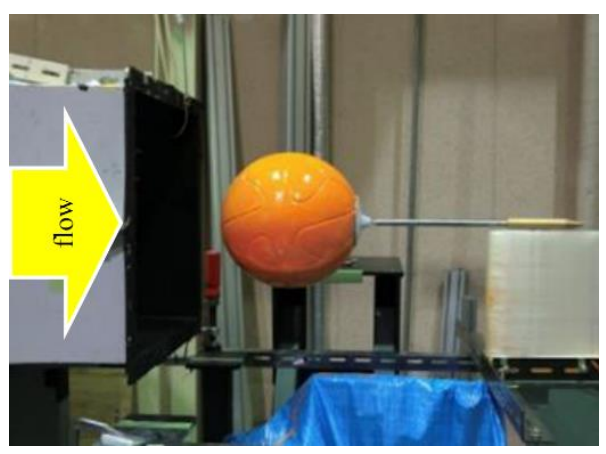

Figure 5. Oil film experiment (Krasava).

\subsection{D-PIV Measurement}

In order to visualize the location of the flow separation point and the flow in the vicinity for the four balls shown in Table 1, 2D-PIV measurements were performed as shown in Figure 6. Three velocity ranges were measured: subcritical region (laminar flow), critical region, and supercritical region (turbulent flow). PIV images were taken with a NAC MEMRECAM HX-3 at 2000 FPS with a resolution of 5 megapixels. A Lee Laser MODEL LDP-100MQG was used as the laser light source, with a peak pulse power of 
$2.0 \mathrm{~kW}$. We used Koncerto II, an analysis software from Seika Digital Image. The laser light sheet was placed on a meridian line of the soccer ball and photographed from the side with a high-speed camera. The high-speed camera's shooting interval was set to $15 \mu \mathrm{s}$, the average frame rate was $2000 \mathrm{fps}$, and the thickness of the laser light sheet was set to $3 \mathrm{~mm}$. The smoke of Dioctyl sebacate was used as tracer particles.

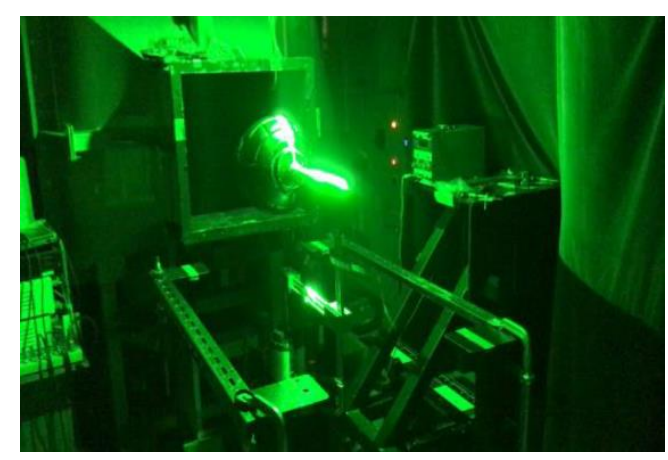

Figure 6. 2D-PIV (particle image velocimetry) measurement.

\section{Results and Discussion}

\subsection{Groove Shape Measurement}

Figure 7a-c show the total length, the cross-sectional area, and the total volume of the panel grooves. For those with multiple types of grooves, such as Ordem, Teamgeist, and Merlin, the length and cross-sectional area were measured separately. Ordem, Merlin, and Finale have relatively longer groove lengths and larger groove volumes. On the other hand, Delta, Teamgeist, and Jabulani have a shorter length and a smaller cross-sectional area of grooves, resulting in a smaller total groove volume. Thus, the length and volume of the grooves vary several times, depending on the ball type.

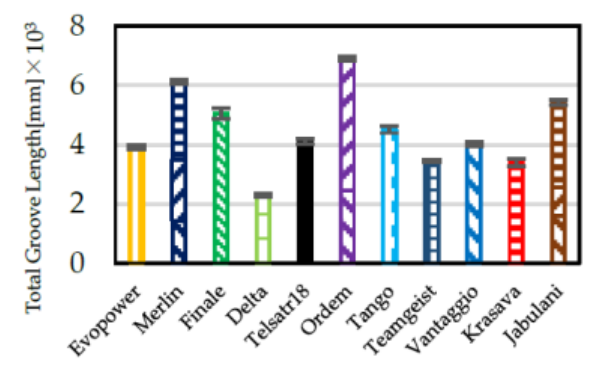

(a) Total grove length

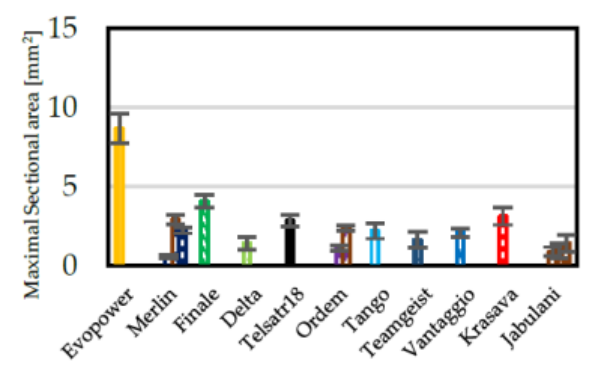

(b) Cross section of each groove

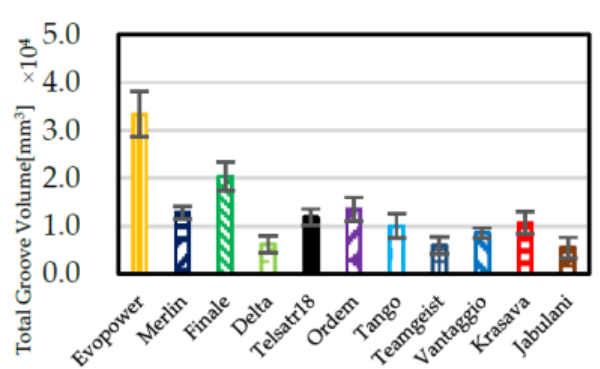

(c) Total groove volume

Figure 7. Specifications of ball groove.

\subsection{Fluid Force Experiment}

Figure 8 shows the $C_{D}$ diagram for each ball. The drag coefficients of all the balls are large in the laminar flow region where the Reynolds number is low, but in the turbulent area where the Reynolds number is high, a drag crisis occurs, and the drag coefficient becomes small. In the recently released Merlin, the Reynolds number at which the drag crisis occurs is smaller than in other balls. The drag coefficients in the laminar and turbulent regions were relatively more extensive than those of the other balls. As shown in Figure 8, Merlin was characterized by a more considerable panel groove length and volume than the other balls. This larger total volume of the grooves has affected the drag crisis and drag coefficient. The changes in the drag coefficient of the other balls were consistent with the trends shown in previous studies $[8,9,12,21-23]$. Thus, the drag coefficient dropped rapidly from about 0.5 to 0.2 in the Reynolds numbers range from $1.0 \times 10^{5}$ to $2.4 \times 10^{5}$. This drag 
crisis corresponds to a change in the aspect of the flow around the ball from laminar to turbulent, but the Reynolds number at which the drag crisis occurs varies significantly from ball to ball. Therefore, we focused on the first and the last Reynolds numbers of the drag crisis to characterize these phenomena. The first and last Reynolds numbers of the drag crisis are smallest at $1.0 \times 10^{5}$ and $1.2 \times 10^{5}$ for Evopower and largest at $1.8 \times 10^{5}$ and $2.4 \times 10^{5}$ for Jabulani. The Reynolds number between the start and endpoints of the drag crisis, the critical region's width, is about 0.3 for Evopower and about 0.6 for Jabulani, which is also a significant difference between the balls.
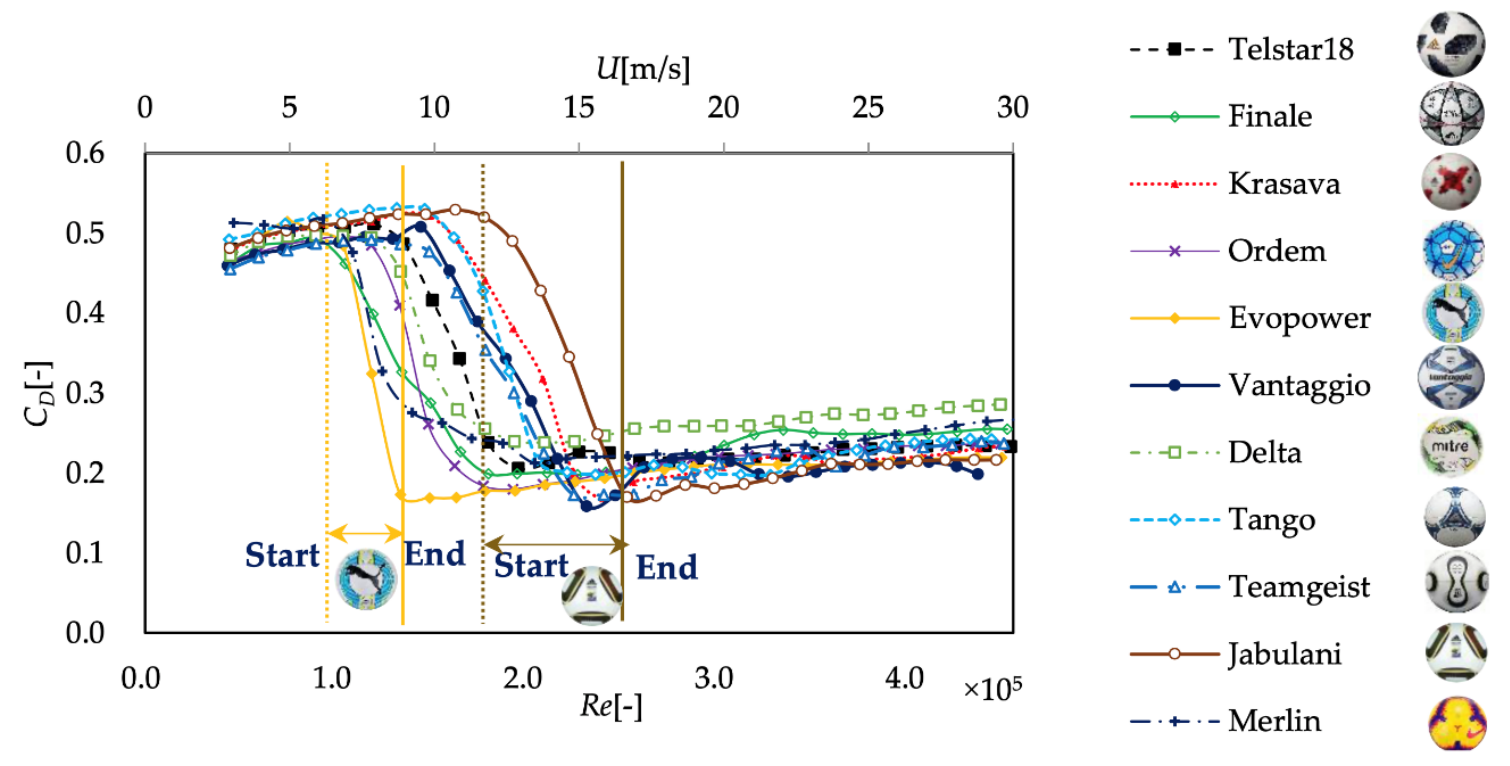

Figure 8. The drag coefficient $\left(C_{D}\right)$ diagram of each ball.

Figures 9 and 10 show the relationship between the volume of the grooves and the Reynolds number to clarify the dependence of the start and end of the drag crisis and the width of the critical region on ball panel geometry. As shown in the figure, there is a strong correlation between the grooves' volume and the Reynolds number of the critical region. The correlation coefficients are -0.63 for the starting Reynolds number and -0.83 for the ending Reynolds number. Figure 11 shows the relationship between the groove volume and the width of the critical region. Overall, the width of the critical region became smaller as the groove volume was increased. However, when Evopower was excluded, there was no clear correlation between the groove volume and the width of the critical region. Thus, the panel grooves significantly impact how the drag crisis occurs, i.e., the transition from laminar to turbulent flow. In order to elucidate the details of this mechanism, we continued to visualize the separation points by oil film experiments and 2D-PIV measurements to investigate how the transition from laminar to turbulent flow is induced. The relationship between groove volume and the drag coefficient in subcritical and supercritical regions is shown in Figures 12 and 13. There is a weak correlation between the groove volume and drag coefficient of 0.40 and -0.20 in each velocity range, respectively. In the subcritical region, where the flow is laminar, the drag coefficient increases with the number of grooves, i.e., the rougher the surface, the higher the drag coefficient. In the turbulent, supercritical region, the drag coefficient decreased as the volume of the grooves increased. This may be due to the degree of accelerated turbulence, but the trend was not very clear. 


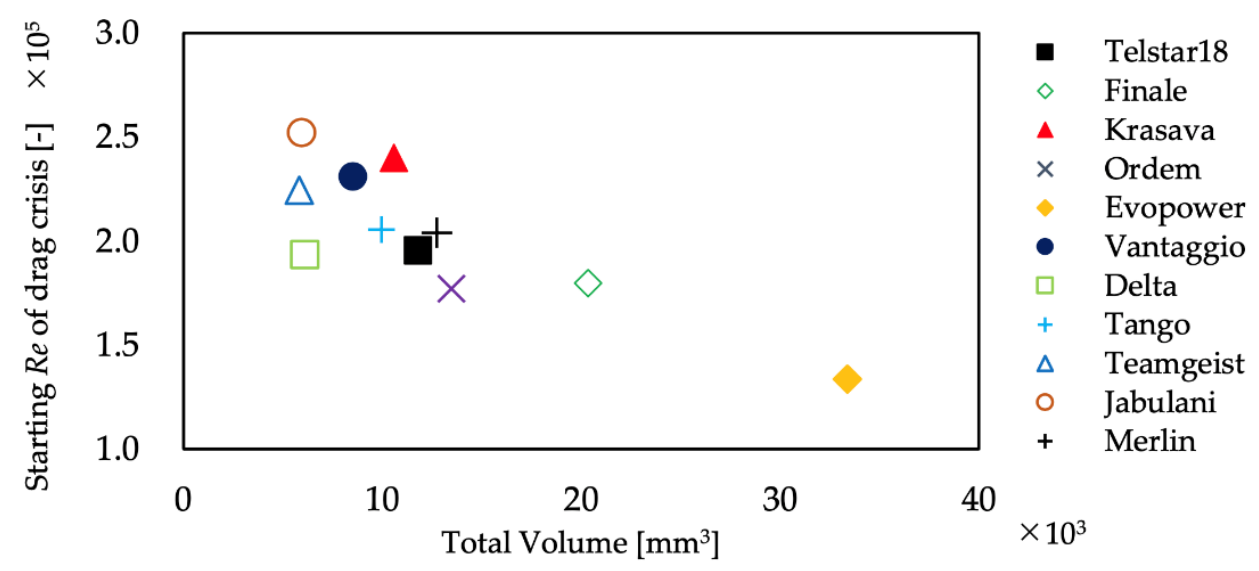

Figure 9. The relationship between the Starting Reynolds number $(R e)$ of drag crisis and total groove volume.

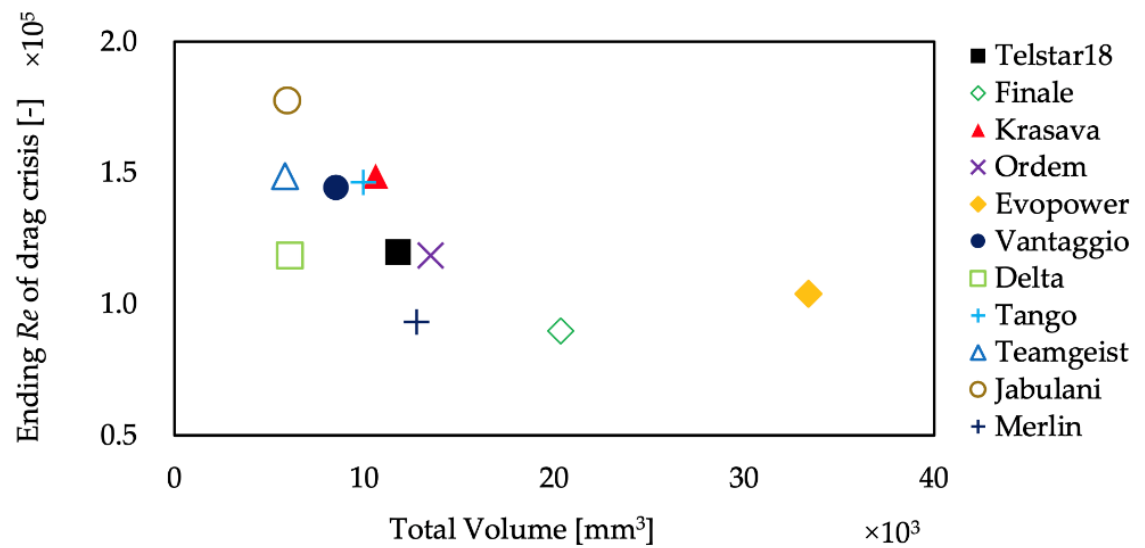

Figure 10. The relationship between the Ending Reynolds number $(R e)$ of drag crisis and total groove. volume.

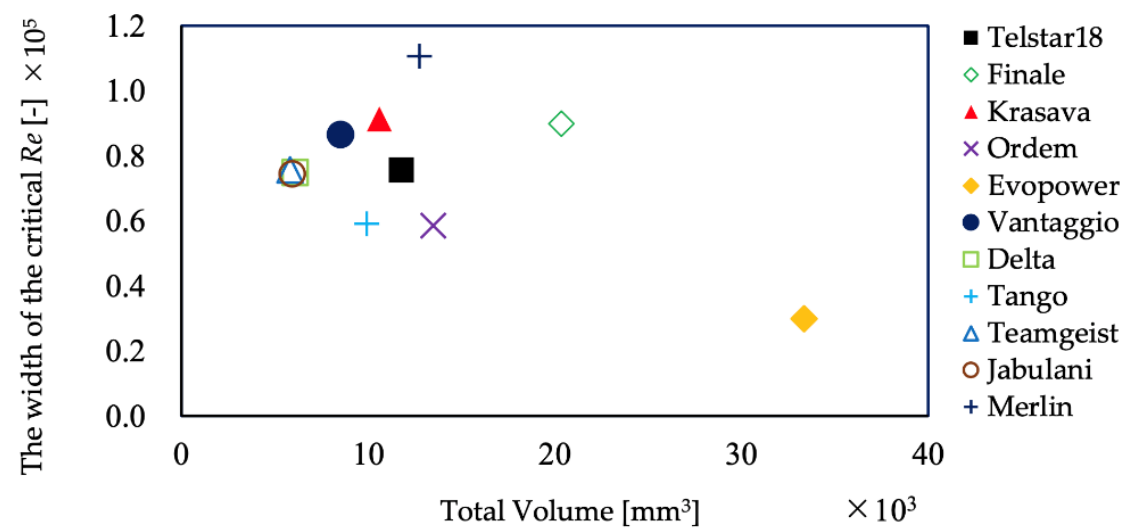

Figure 11. The relationship between the width of the critical region and total groove volume. 


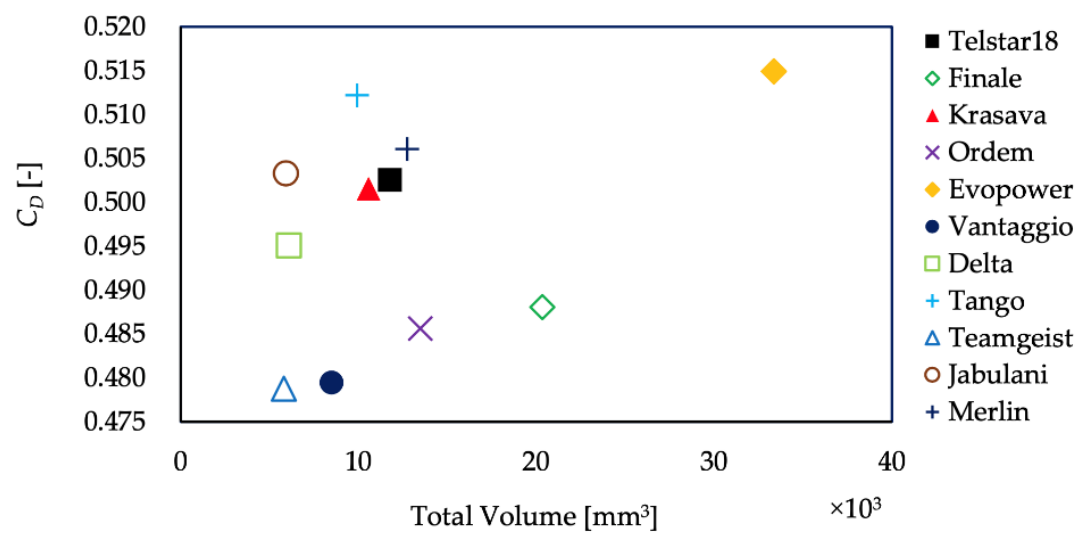

Figure 12. The relationship between groove volume and drag coefficient $\left(C_{D}\right)$ in the subcritical regions.

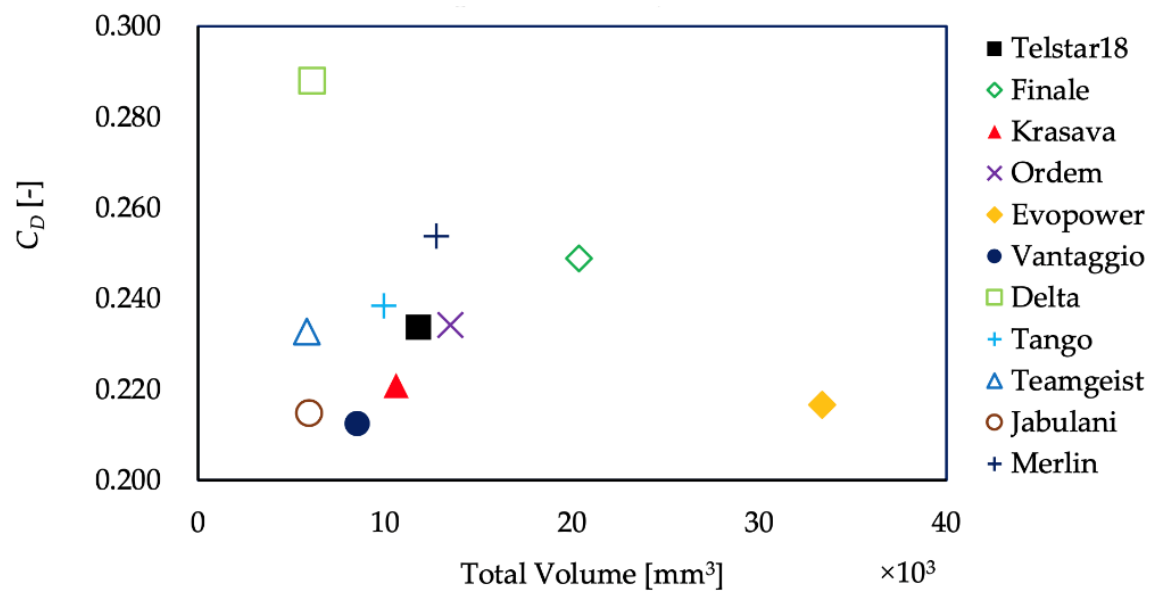

Figure 13. The relationship between groove volume and drag coefficient $\left(C_{D}\right)$ in the supercritical regions.

\subsection{Oil Film Experiments}

Figure 14 shows side views of oil film measurements for (a) a smooth ball, (b) Telstar 18, (c) Krasava, (d) Finale, and (e) Vantaggio. The wind direction was set from left to right. All measurements were performed at supercritical conditions with $\operatorname{Re}=4.3 \times 10^{5}$. In the smooth sphere (a), the first and second separation points appeared on the surface. This feature is consistent with a previous study [4]. The dashed line shows the line of separation bubbles that reattach to the sphere's surface after the boundary layer has detached. The solid line shows the point at which the flow around the sphere finally separated. As shown in this figure, detachment is delayed in the turbulent flow, indicating that the drag coefficient under the turbulent flow is small. On the other hand, only the turbulent separation line is observed in the soccer ball with panel grooves. This result indicates that the panel grooves have shifted the flow from laminar to turbulent flow. The separation point is nearly the same for all soccer balls, corresponding to the fact that the $C_{D}$ values were almost the same for the drag measurements at a velocity of $29.6 \mathrm{~m} / \mathrm{s}$. 


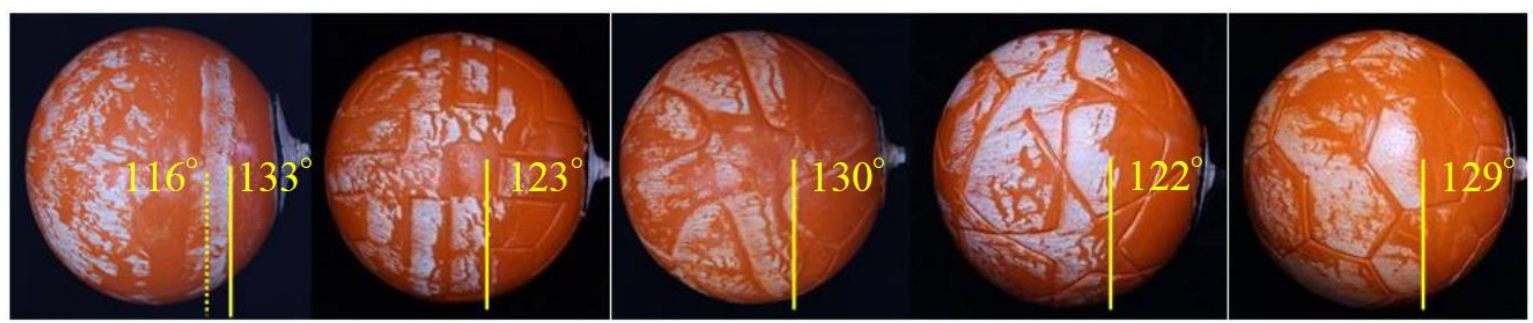
(a) Smooth sphere
(b) Telstar18
(c) Krasava
(d) Finale
(e) Vantaggio

Figure 14. The results of the oil film experiment to show the separation point on the panels.

Figure 15 shows the relationship between the $C_{D}$ values in the supercritical region obtained from the fluid force measurements and the separation position in the oil film experiment. The $C_{D}$ value increases as the separation angle increases, and this correlation coefficient is as large as 0.90 . Therefore, the position of the detachment point changes depending on the shape of the ball groove in the supercritical region, and the drag coefficient changes accordingly.

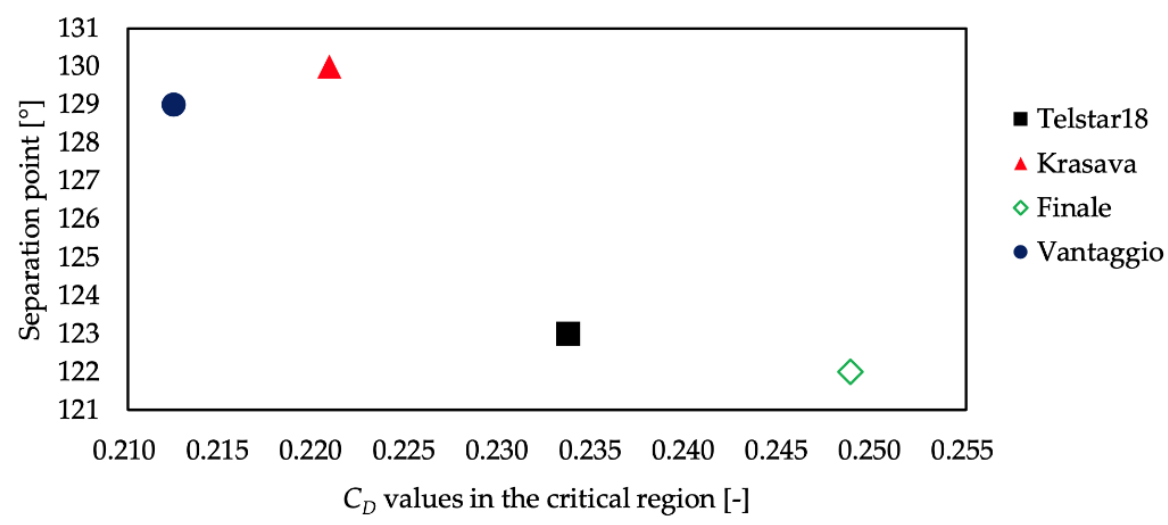

Figure 15. The relationship between the $C_{D}$ values in the supercritical region and the separation position of the oil film experiments.

\subsection{D-PIV Measurement}

In order to clarify the influence of ball panels and grooves in the critical region, 2D-PIV measurements were performed. Tables 2-5 show the results of Evopower, Vantaggio, Jabulani, and Teamgeist measured in the subcritical, critical, and supercritical flow regions. The ball is painted black to reduce the reflection of the PIV laser. The flow around the ball is influenced by the complex three-dimensional groove geometry and is considered to have a three-dimensional structure. Therefore, in order to obtain three-dimensional information about the flow around the ball, the flow was measured at three locations with different positions of the measurement surface. The lines drawn in the pictures of balls $a, b$, and $c$ indicate the plane measured by the PIV. The structure of the surface grooves on each surface depends on the bonding status of the panels. In the images of the PIV measurements, the separation point and flow direction from the separation position are shown as white circles and solid lines. 
Table 2. The PIV results of Evopower with the different panel orientations and flow velocity.

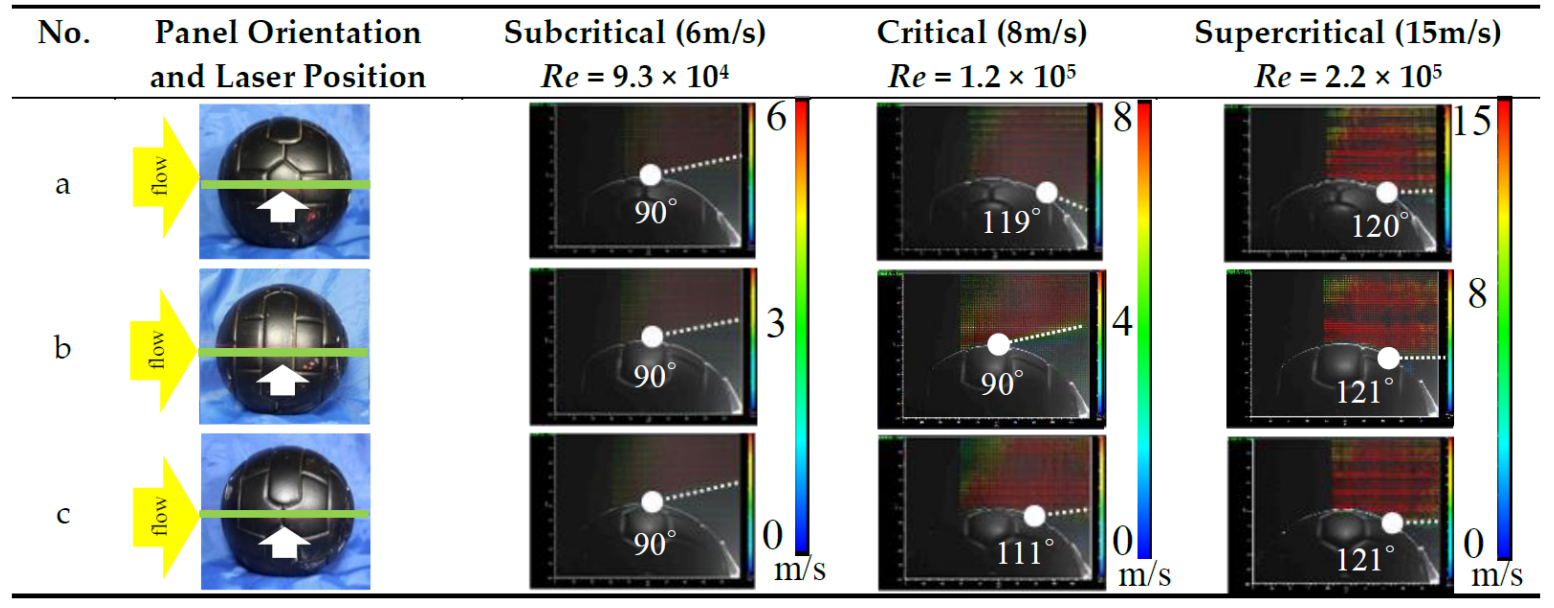

Table 3. The PIV results of Vantaggio with the different panel orientations and flow velocity.

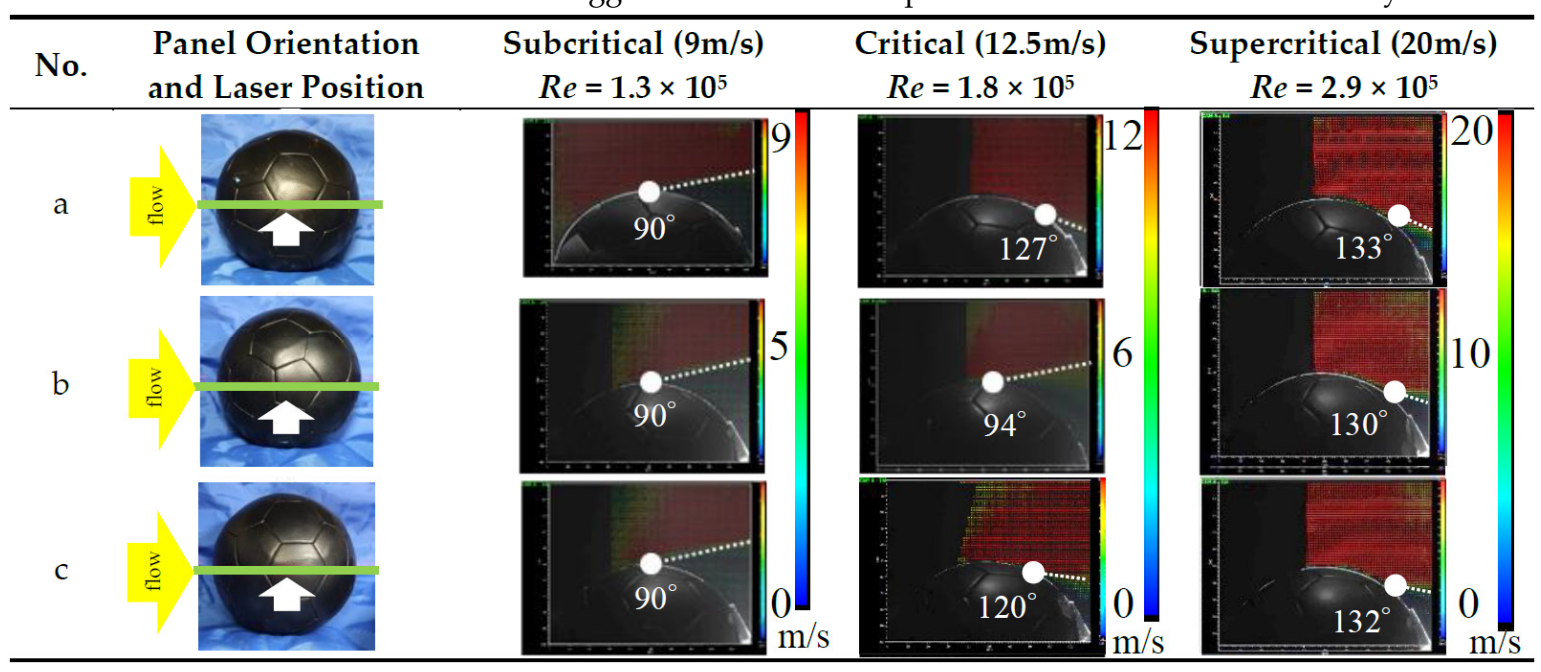

Table 4. The PIV results of Jabulani with the different panel orientations and flow velocity.

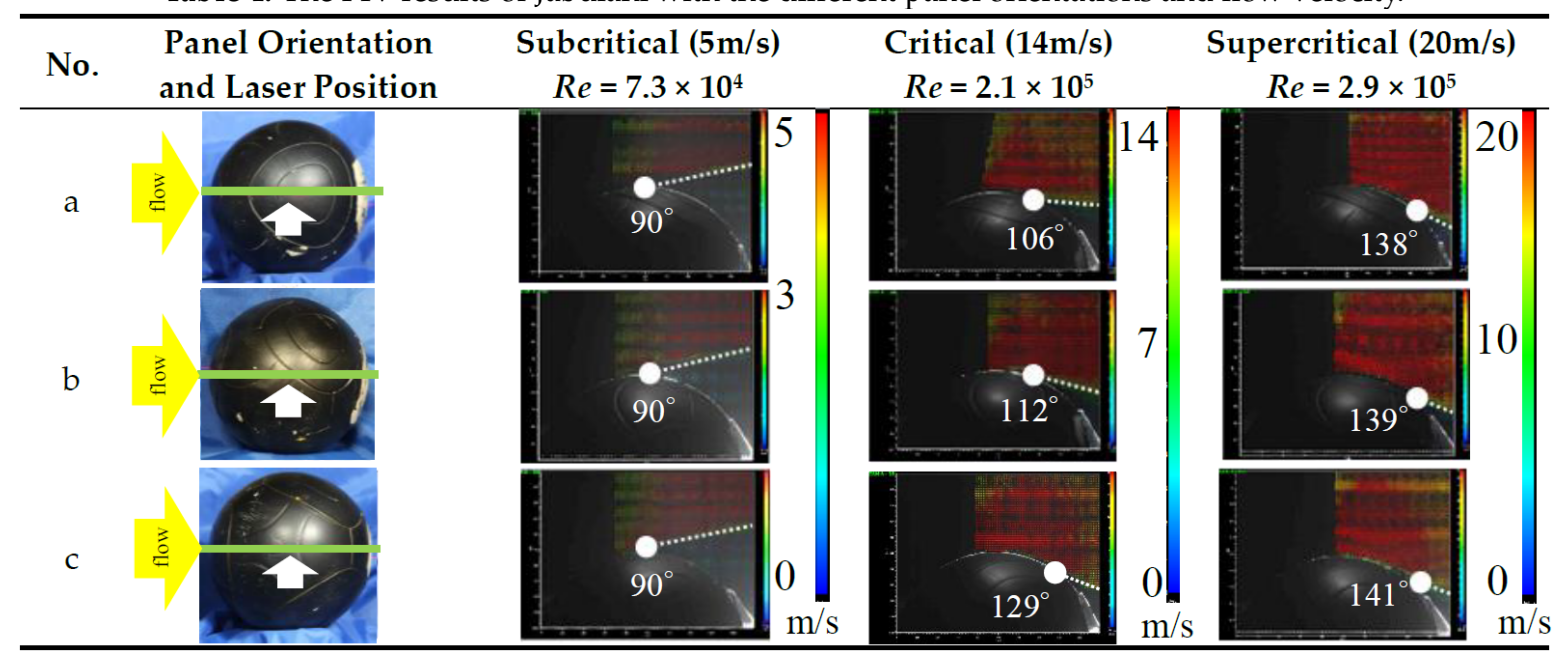


Table 5. The PIV results of Teamgeist with the different panel orientations and flow velocity.

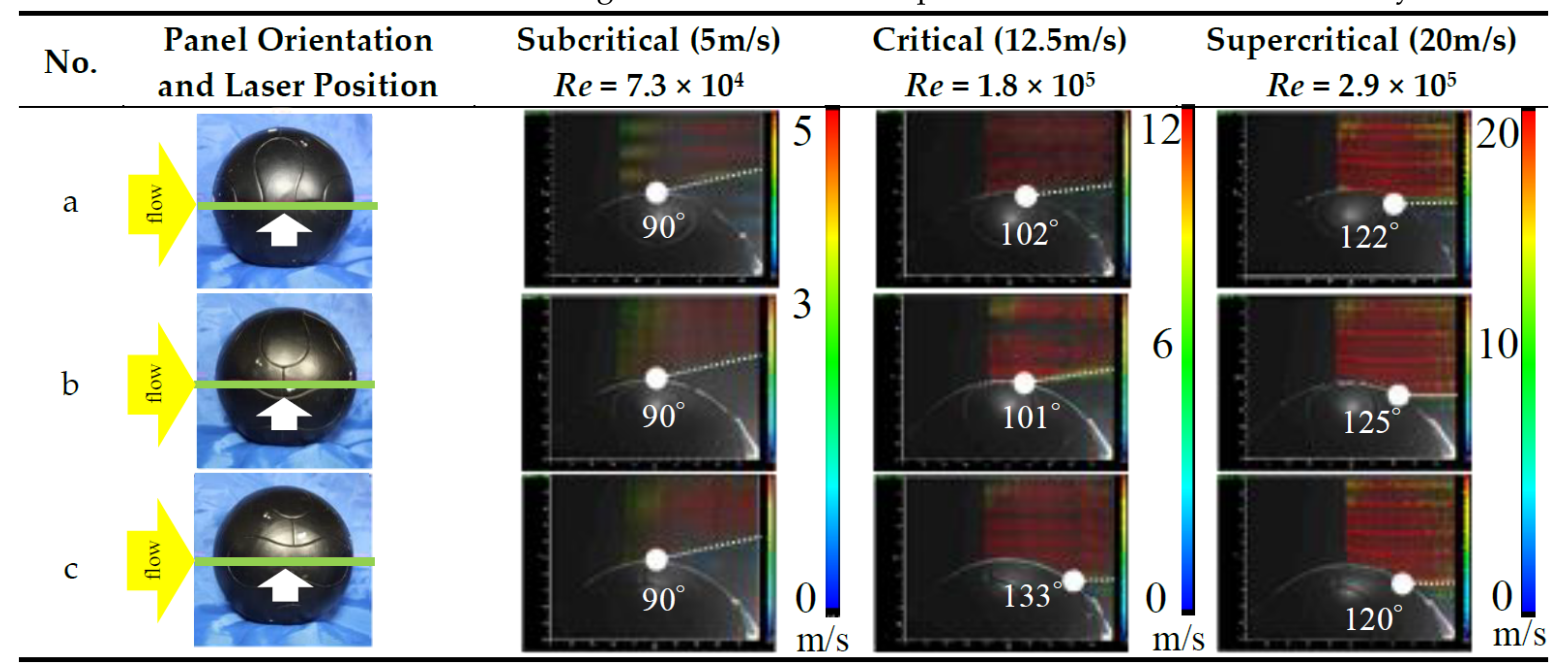

In both the subcritical and supercritical regions, the flow direction at the separation point and the wake were almost the same for the same type of ball in any measurement plane. In the subcritical region, the flow separation occurred at around $90^{\circ}$ for all the balls when the residence point was set to $0^{\circ}$. The $C_{D}$ in this region was relatively large, about 0.5 . The fast separation of the laminar flow increased the width of the wake, which resulted in higher pressure drag.

Next, in the supercritical region, the $C_{D}$ was smaller, about 0.2 , because the flow transitioned to turbulence with the increase of the Reynolds number, and the detachment was delayed. As a result, the wake became narrower, and the pressure drag became small. The location of flow detachment in the supercritical region was generally consistent with the location of the detachment shown by the oil film experiments in Figure 11.

Finally, for the flow in the critical region, the position of the separation point and the direction of the wake varied depending on the position of the measured surface. For example, in the Vantaggio measurement planes a and c in Table 3, the separation position of the critical region flow was behind the ball and showed the same trend as the results for the supercritical region. On the other hand, in panel $b$, the detachment position is about $90^{\circ}$, indicating that the flow is detached in the same way as it is in the subcritical region. Therefore, even for the same ball, depending on the surface properties of the flow path, laminar and turbulent flow regions coexist in the critical region. Similar results were obtained for the other three types of balls. There was no clear correlation between variation in the separation point and the groove volume.

For the soccer ball, the drag reduction in the critical region was slower. Also, the width of the Reynolds number of the drag crisis was wider than it was for the smooth ball. These characteristics are due to the wide range of Reynolds numbers that change from laminar to turbulent flow depending on the position of the ball, as seen in the present results. In addition, in the critical region where laminar and turbulent flows coexist at different positions, the formation of jets is not uniform around the ball. This uneven formation suggests that if the ball is rotating slowly, the direction of the jet would also rotate with the ball and possibly cause an irregular flight path. After the ball is kicked, the drag force reduces the velocity of the flow and decelerates through the critical region to the subcritical region. The non-uniformity of the flow around the ball due to this flow transition is considered to be important to the irregular trajectory of the ball.

On the other hand, it was difficult to determine how the grooves drive turbulence from the present results. For example, in the Jabulani case shown in Table 5, figure c does not appear to be driving turbulence at first glance because the surface is smooth. However, it detaches the slowest of the three measured surfaces. Therefore, it is also indicated that the requirement for turbulence in critical region is not just the number of grooves. In 
order to understand in more detail how the geometry of the groove promotes turbulence, more detailed measurement and analysis is needed, including not only two-dimensional measurements, but also three-dimensional effects. In this study, analysis was based on the flow visualized by PIV and focused on the location of the separation point. In future work, it will be important to quantitatively analyze the wake width of the flow and the flow structure around the groove for a more detailed understanding of flow transitions.

\section{Conclusions}

In order to clarify the relationship between the aerodynamic forces and the shape of the soccer ball panel grooves, measurements of the shape of the grooves, hydrodynamic forces, oil film experiments, and 2D-PIV measurements of the separation point and flow were conducted. As a result, the following results were obtained.

1. The dependence of the drag coefficient of each ball on the Reynolds number was measured. In addition to the magnitude of drag coefficients, the Reynolds number of the drag crisis was different for each panel shape.

2. As the total volume of panel grooves increased, the Reynolds number at which the drag crisis occurred and the Reynolds number at which it ended tended to be smaller.

3. Although smaller than in the critical region, in the laminar and turbulent regions, there was ball dependence and panel dependence on the location of the separation point of the flow.

4. In the critical region, laminar and turbulent flows coexist on the ball, and the separation point of the flows varies greatly depending on the panel surface. Such an uneven flow would affect the formation of the soccer ball's jet and its irregular flight path.

5. Immediately after the ball is kicked, its velocity is large, and it flies in a supercritical state. The ball is slowed down by aerodynamic drag during its flight, but when the total volume of the grooves is large, the supercritical state is more likely to continue, and the $C_{D}$ value and flow conditions do not change much. On the other hand, when the total volume of the groove is small, the critical state is entered earlier, and the $C_{D}$ value and flow conditions change significantly. Therefore, to keep the ball on a stable trajectory, the ball should be designed so that the volume of the grooves is large.

Author Contributions: Conceptualization, S.I.; methodology, S.I.; validation, Y.S., S.I. and M.H.; investigation, Y.S.; data curation, Y.S.; writing-original draft preparation, Y.S. and M.H.; writingreview and editing, M.H.; visualization, Y.S.; supervision, S.I. and M.H.; project administration, S.I.; All authors have read and agreed to the published version of the manuscript.

Funding: This research received no external funding.

Conflicts of Interest: The authors declare no conflict of interest.

\section{References}

1. Passmore, M.; Rogers, D.; Tuplin, S.; Harland, A.; Lucas, T.; Holmes, C. The aerodynamic performance of a range of FIFA-approved footballs. J. Sports Eng. Technol. 2012, 226, 61-70. [CrossRef]

2. Kray, T.; Franke, J.; Frank, W. Magnus effect on a rotating soccer ball at high Reynolds numbers. J. Wind Eng. Ind. Aerodyn. 2014, 124, 46-53. [CrossRef]

3. Alam, F.; Chowdhury, H.; Moria, H.; Fuss, F.K. A comparative study of football aerodynamics. Procedia Eng. 2010, 2, $2443-2448$. [CrossRef]

4. Taneda, S. Visual observations of the flow past a sphere at Reynolds numbers between 104 and 106. J. Fluid Mech. 1979, 85, 187-192. [CrossRef]

5. Murakami, M.; Kondoh, M.; Iwai, Y.; Seo, K. Measurement of aerodynamic forces and flow field of a soccer ball in a wind tunnel for knuckle effect. Procedia Eng. 2010, 2, 2467-2472. [CrossRef]

6. Hiratsuka, M.; Ito, S.; Miyasaka, K.; Konno, A. Stereo three-dimensional particle image velocimetry measurement and aerodynamic force analysis of non-spinning soccer balls. Proc. Inst. Mech. Eng. Part P J. Sports Eng. Technol. 2020, 234, 146-153. [CrossRef]

7. Ito, S.; Kamata, M.; Asai, T.; Seo, K. Factors of unpredictable shots concerning new soccer balls. Procedia Eng. 2012, 34, 152-157. [CrossRef] 
8. Goff, J.E.; Asai, T.; Hong, S. A comparison of Jabulani and Brazuca non-spin aerodynamics. J. Sports Eng. Technol. 2014, 228, 188-194. [CrossRef]

9. Goff, J.E.; Hong, S.; Asai, T. Aerodynamic and surface comparisons between Telstar 18 and Brazuca. J. Sports Eng. Technol. 2018, 232, 342-348. [CrossRef]

10. Hong, S.; Goff, J.E.; Asai, T. Effect of a soccer ball's surface texture on its aerodynamics and trajectory. Proc. Inst. Mech. Eng. Part P J. Sports Eng. Technol. 2019, 233, 67-74. [CrossRef]

11. Hong, S.; Sakamoto, K.; Washida, Y.; Nakayama, M.; Asai, T. The influence of panel orientation on the aerodynamics of soccer balls. Procedia Eng. 2014, 72, 786-791. [CrossRef]

12. Hong, S.; Asai, T. Effect of panel shape of soccer ball on its flight characteristics. Sci. Rep. 2015, 4, 5068. [CrossRef]

13. Alam, F.; Chowdhury, H.; Stemmer, M.; Wang, Z.; Yang, J. Effects of surface structure on soccer ball aerodynamics. Procedia Engineering. 2012, 34, 146-151. [CrossRef]

14. Goff, J.E. A review of recent research into aerodynamics of sport projectiles. Sports Eng. 2013, 16, 137-154. [CrossRef]

15. Hong, S.; Asai, T. Aerodynamic effects of dimples on soccer ball surfaces. Heliyon 2017, 3, e00432. [CrossRef]

16. Goff, J.E.; Carr, M.J. Trajectory analysis of a soccer ball. Am. J. Phys. 2009, 77, 1020-1027. [CrossRef]

17. Myers, T.G.; Mitchell, S.L. A mathematical analysis of the motion of an in-flight soccer ball. Sports Eng. 2013, 16, 29-41. [CrossRef]

18. Mizota, T.; Kurogi, K.; Ohya, Y.; Okajima, A.; Naruo, T.; Kawamura, Y. The strange flight behavior of slowly spinning soccer balls. Sci. Rep. 2013, 3, 1871. [CrossRef]

19. Hong, S.; Asai, T.; Seo, K. Visualization of air flow around soccer ball using a particle image velocimetry. Sci. Rep. 2015, 5, 15108. [CrossRef]

20. Hong, S.; Asai, T.; Seo, K. Flow Visualization Around Panel Shapes of Soccer Ball. Procedia Eng. 2015, 112, 391-394. [CrossRef]

21. Asai, T.; Seo, K. Aerodynamic drag of modern soccer balls. SpringerPlus 2013, 2, 171. [CrossRef]

22. Goff, J.E.; Hobson, C.M.; Asai, T.; Hong, S. Wind-tunnel Experiments and Trajectory Analyses for Five Nonspinning Soccer Balls. Procedia Eng. 2016, 147, 32-37. [CrossRef]

23. Kiratidis, A.L.; Leinweber, D.B. An aerodynamic analysis of recent FIFA world cup balls. Eur. J. Phys. 2018, 39, 034001. [CrossRef] 